\title{
Segmental Patterning of Rat and Chicken Sympathetic Preganglionic Neurons: Correlation between Soma Position and Axon Projection Pathway
}

\author{
Cynthia J. Forehand, ${ }^{1}$ Elizabeth B. Ezerman, ${ }^{1}$ Eric Rubin, ${ }^{2}$ and Joel C. Glover ${ }^{3}$ \\ 'Department of Anatomy and Neurobiology, University of Vermont, Burlington, Vermont 05405, ${ }^{2}$ Department of Biological \\ Psychiatry, New York State Psychiatric Institute, New York, New York 10032, and ${ }^{3}$ Department of Physiology, Institute of \\ Basic Medical Sciences, University of Oslo, 0317 Oslo, Norway
}

The segmental organization of midthoracic rat and chicken sympathetic preganglionic neurons was examined by retrograde labeling in vivo and in vitro. The results demonstrate that individual sympathetic preganglionic neurons project only rostrally or caudally within the sympathetic chain, even though the spinal segment in which they reside provides innervation to both rostral and caudal ganglia. In addition, there is both a segmental and an intrasegmental pattern in the thoracic sympathetic column, in which the position of preganglionic neurons is related to the direction they project in the sympathetic chain. Thoracic sympathetic preganglionic neurons are organized into discrete segmental units, each of which exhibits an internal rostrocaudal polarity with respect to the direction of axon projection in the sympathetic chain. The rostrocaudal bias of this internal polarity is graded from segment to segment along the longitudinal axis. Since there is minimal overlap between thoracic segments, the transition from one segment to another entails a sharp change in the pathway choice of the preganglionic neurons. The organization of the preganglionic projections thus includes (1) segmental periodicity, (2) intrasegmental gradients of neuronal identity, and (3) an axial gradient of segment identity. The significance of these findings is twofold. First, they suggest a functional organization that may be related to the specificity of sympathetic reflex action. Second, they reveal a cellular organization that suggests an underlying segmental pattern in the developing spinal cord.

[Key words: rostrocaudal gradient, spinal cord, neuromere, autonomic nervous system, dextran amines, embryogenesis]

\footnotetext{
Received Nov. 17, 1992; revised June 23, 1993; accepted June 30, 1993.

We thank Tracy Barret, Viktor Ballada, Patricia Newton, and Håvard Tønnesen for expert technical assistance, and Ms. Heather Matthews for participation in some of the experiments. Drs. J. Jansen, K. Leistal, J. Mæhlen, A. Njå, D. Purves, and $\mathrm{M}$. Shankland provided helpful comments on the manuscript. This work was supported by NSF Grants BNS 8518844 and 9120947 and NIH Grant NS 01344 (to C.J.F), and grants from the Norwegian Medical Research Council and the Nansen Fund (to J.C.G.). A grant from the NATO Foundation for International Collaboration (CRG 890641) supported travel between the University of Vermont and the University of Oslo. A portion of this work was accomplished while C.J.F. and E.R. were at Washington University in St. Louis. That portion was supported by NIH Grant NS 18629 to Dr. D. Purves and a Summer Research Fellowship from the University of Rochester School of Medicine and Dentistry (to E.R.)

Correspondence should be addressed to Dr. C. J. Forehand at the above address.

Copyright (C) 1994 Society for Neuroscience $0270-6474 / 94 / 140231-11 \$ 05.00 / 0$
}

It was recognized in the last century that functional specialization along the neuraxis is correlated with an early morphogenetic subdivision of the neural epithelium. Direct observation showed that the rostral neural tube exhibits distinct vesicular expansions that give rise to the forebrain, midbrain, and hindbrain. Even finer anatomical subdivisions, often of about a segment in length, were observed in the embryonic hindbrain and spinal cord, leading to the suggestion that the caudal neural tube is metameric (reviewed in Keynes and Lumsden, 1990). Until recently, it was unclear whether such neuromeres are intrinsic to the neural tube or arise through mechanical deformation by surrounding tissues. However, immunohistochemistry, axon tracing, cell-lineage tracing, and molecular genetic techniques now suggest that at least some of the neuromeres originally proposed on morphological grounds are true segmental compartments (Lumsden, 1990). The evidence is clearest in the hindbrain, where the neuromeres (termed "rhombomeres") are delineated by segmental patterns of cell surface molecules (Lumsden and Keynes, 1989), cell density and proliferation (Guthrie et al., 1992), axon distribution (Lumsden and Keynes, 1989), and cell migratory barriers (Fraser et al., 1990). Moreover, the rhombomeres can be distinguished by differential patterns of cell migration (Baker and Noden, 1990), axon pathway choice (Glover, 1989), and expression of specific regulatory genes (Wilkinson and Krumlauf, 1990). Although the mechanisms by which such metameric units are established are not yet known, it is clear that rhombomeres have a genetic, molecular, and cellular basis that resides within the neural tube.

The possibility of a similar neuromeric organization in the spinal cord has received less support. The most obvious segmental feature of the spinal cord, the iterated series of spinal nerves and ganglia, is not intrinsic to the neural tube, but arises because these peripheral appendages of the cord are forced to conform to the segmental pattern of the adjacent somitic mesoderm (Keynes and Stern, 1988). An evaluation of early patterns of cell proliferation and neuronal differentiation has revealed no evidence of segmentally iterated cell populations or neuromeric boundaries in the chicken embryo (Lim et al., 1991). Moreover, regulatory gene expression in the spinal neural tube is typically in rostrocaudal gradients, with no evidence of periodic patterns or restriction to single segments as is seen in the hindbrain.

Despite the lack of evidence on these counts, other evidence indicates that the spinal cord may have a neuromeric organi- 
zation. In the zebrafish, the embryonic spinal cord contains primary motoneurons and interneurons that are segmentally iterated (Westerfield et al., 1986). In higher vertebrates, transplantation experiments have demonstrated autonomous regions of functional specificity in the spinal neural tube. In the chicken embryo, brachial and lumbar regions of the neural tube maintain their functional identities when interchanged (Narayanan and Hamburger, 1971), and lumbar motoneurons maintain their segment-specific patterns of peripheral axon pathfinding despite segmental translocations (Lance-Jones and Landmesser, 1981). Lineage tracing experiments in the chicken embryo have demonstrated segmentally iterated barriers to cell movement in the lumbar neural tube (Stern et al., 1991). Lastly, the regulatory genes that are expressed in multisegmental gradients have sharp rostral boundaries at specific and different spinal segments, at least in the cervical and thoracic regions (Kessel and Gruss, 1990).

To explore further the possibility of metameric organization of the cord, we have examined segmental patterning of the sympathetic preganglionic neurons. In maturity, these neurons exhibit segment-specific connectivity patterns that suggest a capacity to recognize and distinguish target cells on the basis of matching rostrocaudal identities (Purves and Lichtman, 1985). Thus, preganglionic neurons in more rostral segments innervate ganglion cells that innervate more rostral end organs (Langley, 1892, 1894; Njå and Purves, 1977a; Lichtman et al., 1979; Yip, 1986). This segmental matching is expressed during reinnervation of ganglion cells (Njå and Purves, 1977b) and during innervation of ectopically positioned sympathetic ganglia (Purves et al., 1981) or segmental muscle (Wigston and Sanes, 1982, 1985). The topographic specificity of innervation and reinnervation in the sympathetic system indicates that preganglionic axons have stable segment-specific identities that influence their connectivity.

Here, we use retrograde axonal tracing to define more clearly the segmental organization of the sympathetic preganglionic neurons that is correlated with the specific segmental connectivity patterns described above. The basis of this organization is more complex than the simple periodic bundling of the preganglionic axons into segmental roots, a feature shared with spinal motoneurons that depends on the periodicity of the adjacent somitic mesoderm. Rather, the segmentation we show here is manifested by the explicit pathway choices made by the preganglionic axons after leaving the segmental nerves to enter the sympathetic chain: rostrocaudal position of a preganglionic neuron within a segment is reflected in the direction of its axonal growth in the sympathetic chain. On the basis of these observations, we suggest that (1) each thoracic segment of the neural tube exhibits an internal pattern of positional information with a rostrocaudal polarity, and (2) the expression of this internal polarity is graded from segment to segment along the rostrocaudal axis.

Some of these results have been presented in abstract form (Forehand and Rubin, 1986; Ezerman el al., 1990).

\section{Materials and Methods}

\section{Animals}

Initial experiments to define segmental projection patterns of midthoracic preganglionic neurons were performed on adult rats. To determine whether the pattern observed was evident in the earliest projections of these neurons, additional experiments were performed on rat embryos. Finally, to determine the phylogenetic generality of the pattern, and to establish a preparation in which the mechanisms underlying the development of this projection pattern can be assessed experimentally, we examined sympathetic preganglionic projections in the chicken embryo.

Adult Sprague-Dawley rats of either sex, including timed pregnant females, were obtained from Charles River. The day following insemination was designated embryonic day 0 (E0), E1 beginning at midnight. White Italian and White Leghorn chicken eggs were obtained from local suppliers and incubated at $37^{\circ} \mathrm{C}$ in a forced draft incubator.

\section{In vitro preparations}

Pregnant rats were anesthetized with sodium pentobarbital $(50 \mathrm{mg} / \mathrm{kg})$, and embryos were removed by cesarean section into ice-cold artificial cerebrospinal fluid (in mM: $\mathrm{Na}, 149 ; \mathrm{K}, 5 ; \mathrm{Mg}, 1 ; \mathrm{Ca}, 2 ; \mathrm{Cl}, 136 ; \mathrm{H}_{2} \mathrm{PO}_{4}$, $1 ; \mathrm{HCO}_{3}, 24$; glucose, 11), gassed with $95 \% \mathrm{O}_{2}, 5 \% \mathrm{CO}_{2}$. E10 chicken embryos were removed from their eggs into ice-cold physiological saline (in mM: Na, $145.6 ; \mathrm{K}, 5 ; \mathrm{Mg}, 1 ; \mathrm{Ca}, 2 ; \mathrm{Cl}, 149.3 ; \mathrm{H}_{2} \mathrm{PO}_{4}, 1 ; \mathrm{HCO}_{3}, 12$; glucose, 11) gassed with $95 \% \mathrm{O}_{2}, 5 \% \mathrm{CO}_{2}$. All embryos were rapidly decapitated and eviscerated. A dorsal laminectomy was performed and in vitro preparations were maintained as previously described (Glover et al., 1986). Preparations were kept cold until all dissection was complete.

\section{Retrograde labeling of sympathetic preganglionic neurons}

To assess whether individual preganglionic neurons in a given segment projcct both rostrally and caudally in the sympathetic chain, differentiable retrograde tracers were placed on opposite sides of a single chain ganglion that had been isolated from the remaining ganglia (Fig. 1). Thus, preganglionic neurons that project in only one direction would be labeled with only one tracer, while neurons that project in both directions in the chain would be labeled with both tracers. In other experiments, the sharpness of segmental boundaries was assessed by applying the tracers to adjacent segmental ganglia on opposite sides of the cord. Comparisons with the segmental boundaries of intercostal motoneuron pools were made by labeling a sympathetic ganglion on one side and the corresponding intercostal nerve contralaterally.

Adult rat. Sympathetic preganglionic neurons in adult rats were retrogradely labeled in vivo with fast blue (FB; Sigma) and/or 2-diamidino yellow (2DY; Dr. Illing, GmbH and Co., KG, Gross-Unstadt, West Germany). Rats were anesthetized with chloral hydrate $(350 \mathrm{mg} / \mathrm{kg})$, intubated, and respirated. The sympathetic chain was exposed as previously described (Rubin and Purves, 1980). After removing the fat surrounding the ganglion, the chain was cut near its junctures with the adjacent ganglia and the distal ends deflected. Approximately $2 \mu \mathrm{l}$ of $4 \%$ aqueous 2DY was pressure injected through a glass micropipette into the chain on one side of the ganglion and $2 \mu 1$ of $5 \% \mathrm{FB}$ was similarly injected into the chain on the other side. To avoid cross-contamination, the dyes were injected as far from the ganglion as possible. To facilitate labeling, care was taken to sever axons in the chain during each injection. Five minutes after the injections, the dyes were swabbed away with physiological saline, and the surgical opening closed. The animals remained on the respirator until they were responsive to pinch.

Chicken and rat embryos. Sympathetic preganglionic neurons in embryos were labeled retrogradely with fluorescent dextran amines (fluorescein-dextran amine, FDA; rhodamine-dextran amine, RDA; Gimlich and Braun, 1985; Glover et al., 1986). To restrict labeling to neurons in an individual segment, the dorsal and ventral roots adjacent to that segment were transected and the sympathetic chain was cut and deflected on both sides of the ganglion of the intact segment. After waiting 10$15 \mathrm{~min}$ to allow the transected axons to seal, fluorescent dextran amines, recrystallized onto the tips of minutien pins, were injected into the sympathetic chain on either or both sides of the ganglion of the intact segment. The tip of the pin was used to cut through the sympathetic chain as the tracer was applied. In experiments where FDA and RDA were applied on either side of the attached ganglion, 10-15 min was allowed to transpire between injections to allow the axons damaged by the first injection to seal before the second tracer was injected. In some expcrimcnts, intcrcostal motoneurons were labeled to compare segmental boundaries of these neurons with those of the preganglionic neurons. To label intercostal motoneurons, fluorescent dextran amines were injected into intercostal nerves approximately $1 \mathrm{~mm}$ from the spinal cord.

Segment identification in rat embryos was made by counting up from the most caudal rib, which lies at thoracic segment 13 (T13). Segment identification in chicken embryos was made by counting up from the 


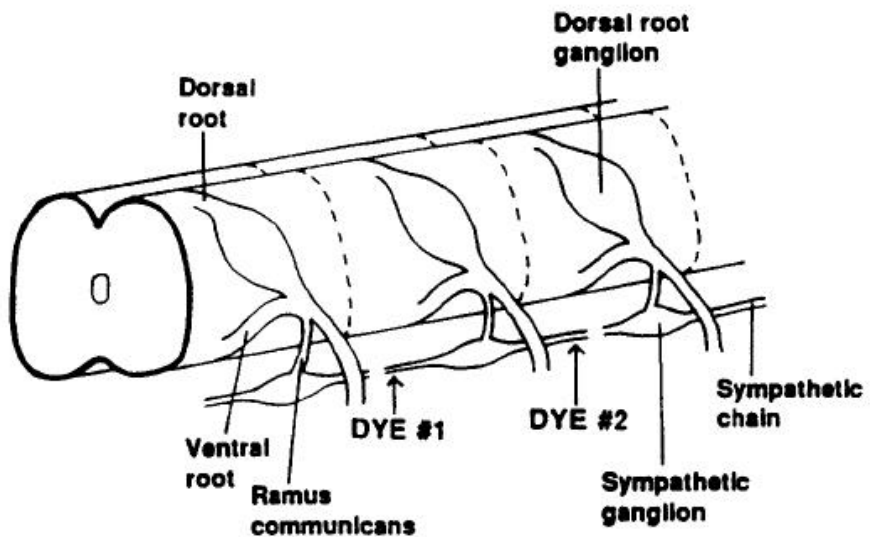

Figure 1. Schematic of the organization of the chicken or rat thoracic spinal cord and paravertebral sympathetic chain ganglia. Each spinal segment is delineated by broken lines. All of the preganglionic neurons within a single segment project out the segmental ventral root and through the ramus communicans into the sympathetic ganglion at the same level. Preganglionic neurons from one segment also provide innervation to more rostral or caudal ganglia; to do this, the axons travel longitudinally within the sympathetic chain. Thus, preganglionic neurons that project rostrally or caudally can be retrogradely labeled by placing dyes (DYE \#1,DYE \#2) on the sympathetic chain rostral or caudal to the segmental ganglion. Severing the sympathetic chain prior to applying the label restricts the label to a single spinal segment.

most caudal of the ribs with ventral struts, which lies at T7. In both species, the sympathetic ganglion of each segment lies just caudal to the corresponding rib. This numbering strategy in the chicken means that $\mathrm{T} 1$ and $\mathrm{T} 4$ as used here would correspond to $\mathrm{T} 2$ and $\mathrm{T} 5$ in the orthograde labeling study of chicken preganglionic neurons by Yip (1990 and personal communication).

\section{Histology and data acquisition}

Adult rat. Two to four days after application of the retrograde tracers, rats were anesthetized with sodium pentobarbital $(50 \mathrm{mg} / \mathrm{kg})$ and perfused transcardially with $0.9 \% \mathrm{NaCl}$ followed by $4 \%$ paraformaldehyde. Spinal cords were removed and postfixed overnight in $4 \%$ paraformaldehyde. The tissue was then equilibrated in 30\% sucrose, frozen in O.C.T. (Miles Laboratory), and cryosectioned transversely at $50 \mu \mathrm{m}$. Serial sections were collected onto subbed slides and coverslipped with buffered glycerin containing $0.1 \%$ paraphenylene diamine to prevent fading of fluorescence. Sections were viewed and photographed with a Leitz Orthoplan fluorescence photomicroscope equipped with a D cube for detection of the FB and 2DY. FB is readily differentiated from the 2DY because the FB fluoresces blue and is cytosolic, while the 2DY fluoresces yellow and is localized to the nucleus.

Chicken and rat embryos. Ten to fourteen hours after application of the retrograde tracers, spinal cords were dissected from the in vitro preparations and fixed in $4 \%$ paraformaldehyde for $2 \mathrm{~d}$, equilibrated in sucrose, frozen in O.C.T., and cryosectioned transversely at $30 \mu \mathrm{m}$. Serial sections were collected onto subbed slides and coverslipped with Citifluor (U. College, London) to prevent fading of fluorescence. Sections were viewed and photographed with a Zeiss fluorescence photomicroscope equipped with Zeiss filter sets $\mathbf{4 8 7 7 1 5}$ and 487717 for rhodamine and fluorescein, respectively.

\section{Data analysis}

Retrogradely labeled neurons were counted manually and tallied in serial sections. Statistical analysis and graphical representation of data were performed using Microsoft EXCEL, KALEIDAGRAPH, and CANVAs programs on a Macintosh computer. To compare and average data from different preparations in which neurons in the same individual segment were labeled, the midpoints of the longitudinal distributions of the labeled neurons in each preparation were aligned and averages and standard deviations of the number of rostrally or caudally projecting cells were calculated on a section-to-section basis.
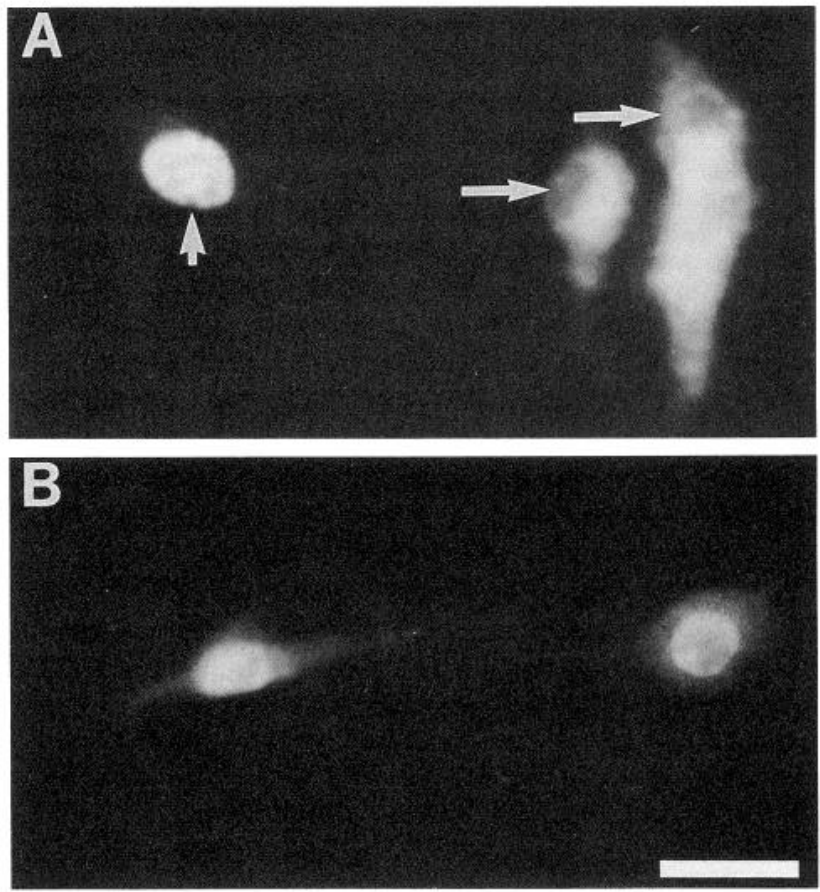

Figure 2. Preganglionic neurons in the adult rat project either rostrally or caudally within the sympathetic chain. $A$, Preganglionic neurons in T6 are labeled with FB placed on the sympathetic chain just rostral to the sixth ganglion and with 2DY placed just caudal to the same ganglion. Individual preganglionic neurons contain only one of the dyes and thus project in only one direction. FB-labeled cells exhibit cytosolic labeling and clear nuclei (long arrows), while 2DY-labeled cells exhibit nuclear labeling (short arrow) and clear cytosol. $B$, In control experiments where both dyes were placed on the same spot on the sympathetic chain, all cells are double labeled as evidenced by both cytosolic and nuclear labeling. Scale bar, $20 \mu \mathrm{m}$.

\section{Results}

Sympathetic outflow from the spinal cord is carried by the axons of preganglionic neurons that are located in a longitudinal column in the intermediate gray. The preganglionic neurons project ipsilaterally to the periphery through the adjacent segmental ventral roots and into the spinal nerves before forming the sympathetic rami to enter the sympathetic chain (see Fig. 1). The set of preganglionic neurons projecting out a single ventral root is operationally defined as a segmental set of the sympathetic preganglionic column; these segmental sets are distributed serially along the longitudinal axis of the spinal cord in register with the ventral roots (i.e., segmental axons always exit from the corresponding segmental root; Rubin and Purves, 1980). The preganglionic neurons innervate ganglion cells at their level of origin and turn into the chain to innervate cells in more distant ganglia.

Electrophysiological investigations in mammals have shown that sympathetic outflow from the thoracic spinal cord is bidirectional (Lichtman et al., 1980). Individual sets of preganglionic neurons at rostral thoracic levels project rostrally in the sympathetic chain while individual segmental sets at caudal thoracic levels project caudally in the chain. Individual segmental sets at intermediate thoracic levels project both rostrally and caudally, with the dominant direction of outflow shifting progressively from rostral to caudal as a function of segmental level. This graded pattern of outflow is correlated with the pattern of innervation of the target sympathetic ganglia: innervation 
Figure 3. Relationship of T7 preganglionic soma positions to direction of axon projection in the adult rat. $A$, Histogram of the number of preganglionic neurons projecting either rostrally or caudally within the sympathetic chain as a function of distance along the rostrocaudal axis of the segment in one experiment. $B$, The percentage of $\mathrm{T} 7$ preganglionic neurons that project caudally increases as a function of increasing caudal position along the rostrocaudal axis.

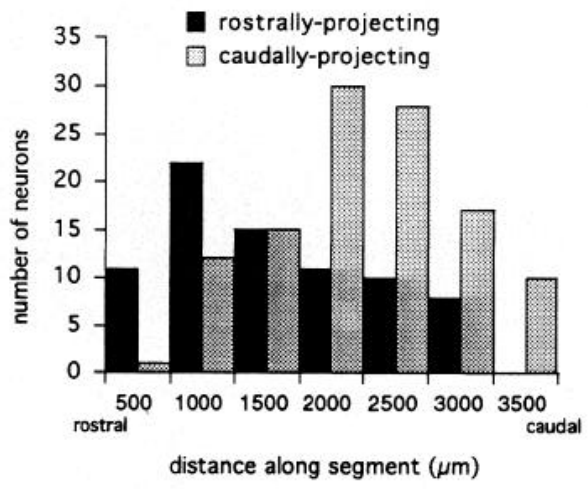

to a given ganglion is strongest from the one or two nearest thoracic segments and decreases in strength when originating from progressively more distant segments (Lichtman et al., 1980).

\section{Origin of rostral and caudal preganglionic projections}

The bidirectional outflow from individual thoracic segments could arise either because individual preganglionic neurons project both rostrally and caudally or because separate neuron populations project either rostrally or caudally. An earlier electrophysiological study in the guinea pig indicated that individual neurons may project in both directions (Lichtman et al., 1980); however, this study did not directly test branching of single axons by antidromic collision. Thus, the possibility exists that their results could be explained by current spread from the stimulating electrode and/or the presence of sensory afferents to ganglion cells projecting within the sympathetic chain. To address directly the question of whether individual preganglionic axons bifurcate to project both rostrally and caudally, we labeled the sympathetic chain rostral and caudal to individual thoracic sympathetic ganglia of the adult rat with different retrograde tracers. The dyes used, FB and 2DY, allowed us to discriminate neurons projecting in only one direction (hence, single labeled) from neurons projecting in both directions (double labeled). Fewer than $3 \%$ of the neurons projected both ros- trally and caudally ( $n=1405$ cells in four preparations) (Fig. 2). Thus, the vast majority of individual preganglionic axons either ascend or descend in the sympathetic chain, but do not branch to project in both directions.

Mapping the distribution of rostrally and caudally projecting neurons within individual mid-thoracic ganglia (T5, T6, or T7) revealed a striking spatial segregation. While the two populations overlapped, rostrally projecting neurons clearly predominated in the rostral portion of the segment and caudally projecting neurons predominated in the caudal portion of the segment (Fig. 3A). The segregation of rostrally and caudally projecting neurons along the longitudinal axis represented a topographic relationship between soma position within the segment and axon trajectory in the sympathetic chain (Fig. 3B).

\section{Position-specific projection patterns arise during embryogenesis} The predominance of neurons that project rostrally or caudally, but not both, could arise by various mechanisms. As an initial step in addressing this issue, we examined the sympathetic preganglionic projection pattern in the rat embryo. In addition, to determine the generality of the pattern, and to establish a preparation in which the mechanisms underlying the development of this projection pattern can be more easily studied, we examined sympathetic preganglionic projections in the chicken
Figure 4. Relationship of T6 preganglionic soma positions to direction of axon projection in the E15 rat. $A$, The number of cells projecting rostrally or caudally is plotted as a function of distance along the rostrocaudal axis of the segment. The bins represent data from consecutive $30 \mu \mathrm{m}$ sections through the spinal cord. Cells that project rostrally are shown above the zero axis while those that project caudally are shown below the zero axis. The mean cell number is indicated by the solid bars and the SD by the shaded bars $(n=3$ preparations). $B$, The percentage of preganglionic neurons that project caudally increases as a function of increasing distance along the rostrocaudal axis.

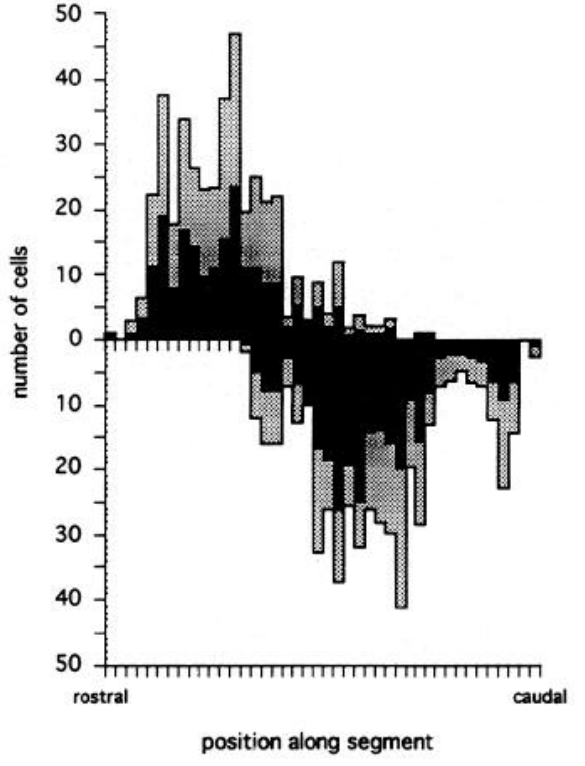

E15 RAT

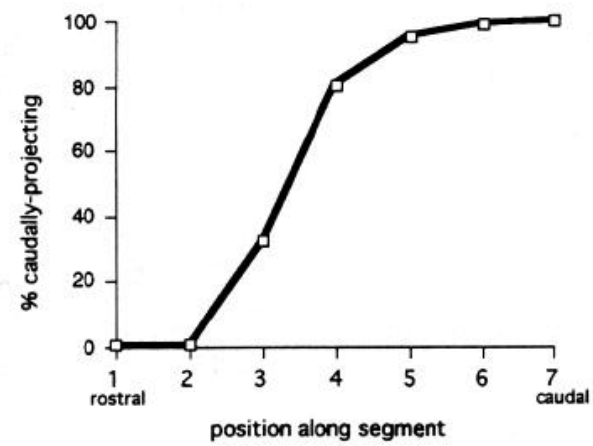



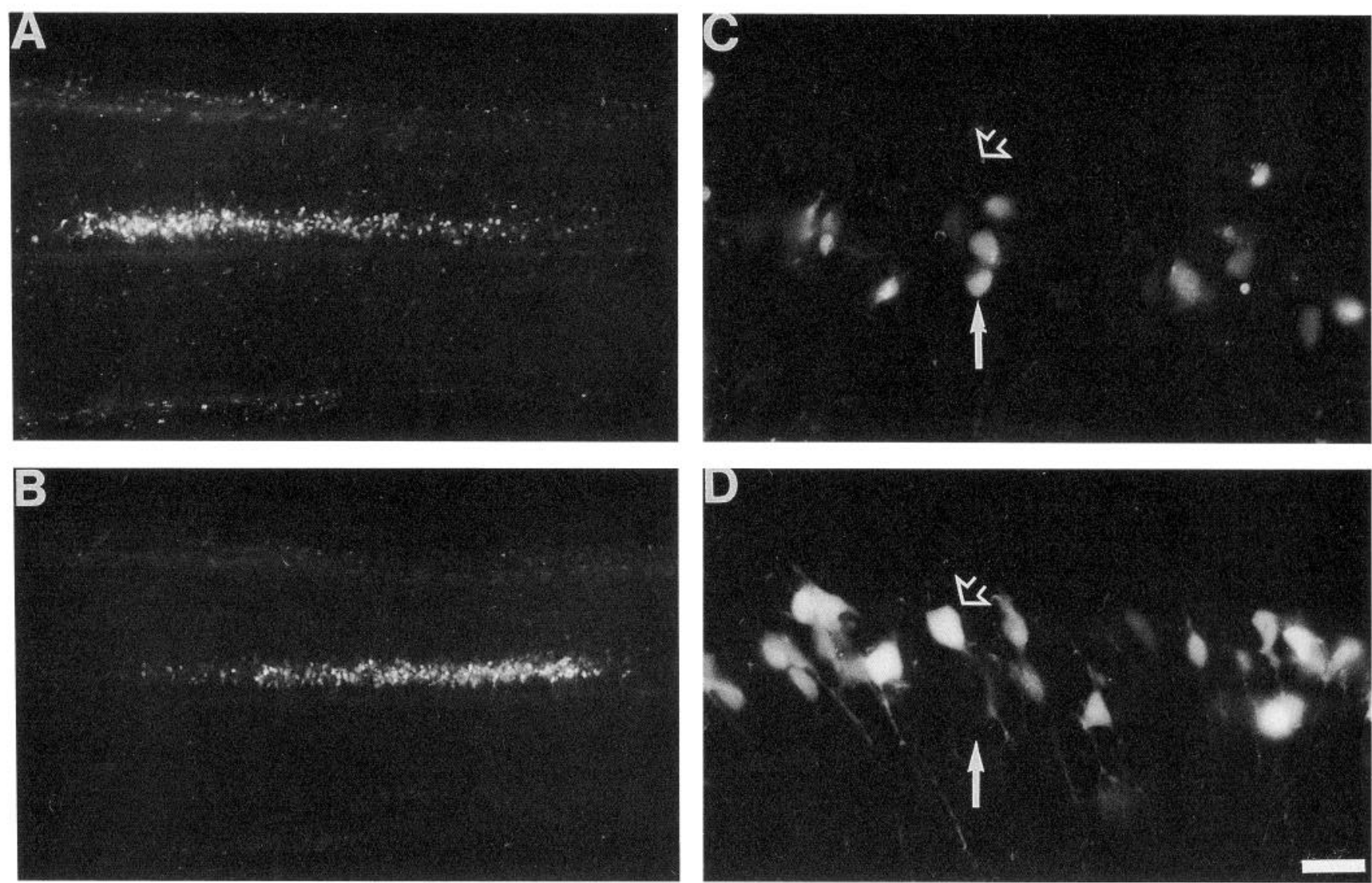

Figure 5. Preganglionic neurons in the E10 chicken embryo project either rostrally or caudally within the sympathetic chain. $A$, Preganglionic neurons in T4 are labeled with FDA that had been placed on the sympathetic chain just rostral to the fourth sympathetic ganglion. $B$, Preganglionic neurons in T4 of the same preparation are labeled with RDA that had been placed just caudal to the fourth ganglion. Within the spinal segment, the majority of FDA-labeled cells ( $A$, photograph taken with fluorescein optics) are located rostral to the majority of RDA-labeled cells ( $B$, photograph taken with rhodamine optics). The photographs represent the same field of a $200 \mu \mathrm{m}$ horizontal section through T4 at the level of the column of Terni. There is a relatively large area of overlap between rostrally and caudally projecting preganglionic neurons within the segment; however, as indicated by examination with high magnification in $30 \mu \mathrm{m}$ sections, double-labeled cells are rare $(C$ and $D)$. $C$, T4 preganglionic neurons labeled with RDA that had been placed on the sympathetic chain just rostral to the fourth sympathetic ganglion. A vertical row of three RDA-labeled neurons is indicated by the solid arrow; these neurons are not labeled with FDA $(D) . D$, T4 preganglionic neurons in the same preparation are labeled with FDA that had been placed just caudal to the fourth ganglion. The open arrow indicates an FDA-labeled neuron that is not labeled with RDA $(C)$. The photographs represent the same field of a $30 \mu \mathrm{m}$ horizontal section through T4 at the level of the column of Terni. Scale bar: $A$ and $B, 100 \mu \mathrm{m} ; C$ and $D, 20 \mu \mathrm{m}$.

embryo. In these experiments, we labeled the sympathetic chain rostral and caudal to individual thoracic ganglia shortly after their coalescence into discrete segmental clusters. In vitro preparations were required because of the inaccessibility of the sympathetic chain in utero and in ovo. Fluorescent dextran amines were used as retrograde tracers because the limited survival time of in vitro preparations precludes the use of tracers such as FB and $2 \mathrm{DY}$.

Rat embryo. Coalescence of thoracic sympathetic ganglia is obvious by E11 in the rat embryo (Rubin, 1985). On E15, it was possible to restrict labeling to a single segment by cutting adjacent ventral roots and cutting and deflecting the sympathetic chain rostral and caudal to the ganglion of interest. The pattern of sympathetic preganglionic projections at E15 was assessed at level T6, and was similar to that seen in the adult (Fig. 4). Rostrally projecting neurons were restricted to the rostral twothirds of the segment, while caudally projecting neurons were restricted to the caudal two-thirds of the segment. Less than $1 \%$ of the neurons ( 2 of 1334 labeled neurons in three experiments) bifurcated in the sympathetic chain to project in both directions.
Chicken embryo. The coalescence of thoracic sympathetic ganglia in the chicken embryo begins by E4; at E10, individual ganglia could be readily recognized in each of the seven thoracic segments, and labeling could be restricted to a single segment as described above. As a first step in comparing avian and mammalian projection patterns, we examined the preganglionic outflow from thoracic segments T2-T6 of the chicken embryo. At E10, preganglionic outflow from a given segment contributed axons to the sympathetic chain both rostral and caudal to the segmental ganglion; however, individual preganglionic neurons with axons that branched to project in both directions were rare (32 of 8310 labeled cells in 17 experiments, $\sim 0.4 \%$ ) (Fig. 5). As has been described in a variety of mammals, preganglionic outflow in the chicken embryo gradually shifted from predominantly rostral in the most rostral thoracic segments to predominantly caudal in the most caudal thoracic segments (Fig. 6). Thus, although chickens have only seven thoracic segments as opposed to the 13 segments of rats, the transition from predominantly rostral to predominantly caudal projections is similarly accomplished in a gradient spanning the thoracic region. 


\section{E10 CHICKEN}

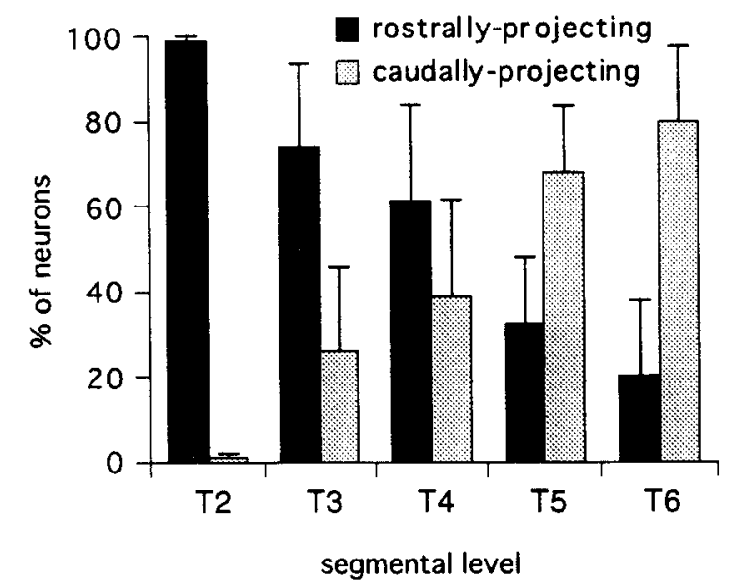

Figure 6. The percentage of E10 chicken preganglionic neurons that project rostrally or caudally changes as a function of segmental level. Values shown are the mean $\pm \mathrm{SD} ; n=3$ preparations at each segment.

Mapping the distribution of rostrally and caudally projecting preganglionic neurons in each individual segment revealed a spatial segregation also similar to that seen in the rat. In those segments containing both types of neuron, the distributions of rostrally and caudally projecting neurons were skewed rostrally and caudally, respectively (Figs. 5, 7). Since all but the most rostral and caudal of the seven thoracic segments were included in this analysis, the transition along the longitudinal axis from predominantly rostral to predominantly caudal outflow is apparent (Figs. 6, 7). As in the rat, there was a topographical relationship between soma position and axon trajectory in each segment (Fig. 8). The proportion of neurons projecting caudally increased progressively as a function of rostrocaudal position. Moreover, this increase changes progressively with successively caudal segments, such that the distribution of rostrally or caudally projecting neurons is characteristic for each segment (Figs. 7,8).

In Figures 4 and 7, the distributions of rostrally and caudally projecting neurons from two to four preparations have been averaged section by section to generate the intrasegmental histograms. Because there are no reliable landmarks at these stages with which to align individual segments from different preparations, the distributions were simply centered on their medians prior to averaging, as shown in Figure 7 for the three individual preparations in which T4 was labeled in the chicken embryo. This method of averaging introduces some variation that could have been minimized had we attempted to align the segments by subjectively comparing their modes. Additional experimental variation certainly originates from differences among preparations in the completeness of labeling. Furthermore, there is no reason to exclude the possibility of real biological variation among embryos. Despite these sources of variation, the systematic segment by segment shift in the intrasegmental pattern is unmistakable (Figs. 7, 8).

\section{Segment boundaries represent a transition in polarity of preganglionic projections}

The topographic relationship between soma position and axon trajectory is such that (1) each thoracic segment is polarized, with a progressively greater propensity for caudal projection by cells located more caudally within the segment, and (2) there is a transition at each segmental boundary from caudally projecting neurons to rostrally projecting neurons. However, in the labeling experiments described above, we only labeled one segment, and could not evaluate the extent to which adjacent segments overlap. To determine the sharpness of the segmental boundaries, preganglionic neurons projecting out adjacent segmental roots were differentially labeled. To do this, the dyes were placed in two consecutive sympathetic chain ganglia on opposite sides of the spinal cord. On each side, the labeled ganglion was isolated from adjacent ganglia by sectioning and deflecting the sympathetic chain rostral and caudal to the ganglion. In eight preparations in which the potential segmental overlap between the third and fourth thoracic ganglia was assessed in $30 \mu \mathrm{m}$ transverse sections through the cord, the overlap was $\leq 116.3 \pm 10.5 \mu \mathrm{m}$. The relative sharpness of this boundary is apparent in horizontal sections through the preganglionic cell column (Fig. 9A). To be certain that no bias was introduced by labeling the consecutive ganglia on opposite sides of the spinal cord, the symmetry of the segmental restriction of preganglionic neurons was demonstrated by labeling left and right ganglia at the same level. The rostral and caudal segmental boundaries were equivalent for left and right preganglionic pools with a spread similar to that observed between segments (Fig. 9B).

The sharpness of the boundaries between segmental blocks of preganglionic neurons prompted a comparison with the segmental arrangement of motoneurons in the thoracic region. To do this, an individual ganglion and the corresponding ipsi- or contralateral intercostal nerves were labeled (Fig. 9C). The concordance between rostral and caudal segmental boundaries of preganglionic versus intercostal motor pools was similar to that seen between bilateral preganglionic segments. An independent assessment of overlap between adjacent segmental intercostal motor pools also indicates a similar overlap of approximately $70 \mu \mathrm{m}$ in the E10 chicken (V. Stirling and J. Glover, unpublished observations).

\section{Discussion}

This study demonstrates that the sympathetic outflow that is directed rostrally versus caudally in the sympathetic chain originates from separate populations of preganglionic neurons. In addition, there is both a segmental and an intrasegmental pattern in the thoracic sympathetic column, in which the position of preganglionic neurons is related to the direction they project in the sympathetic chain. The significance of these findings is twofold. First, they suggest a functional organization that may be rclated to the spccificity of sympathetic reflex action. Second, they reveal a cellular organization that suggests an underlying segmental pattern in the developing spinal cord.

\section{Functional implications}

The functional organization of the sympathetic nervous system allows for the specific activation of target cells based on topography or type, as well as the coordinate activation of target cells that underlies global responses (Cannon, 1915; Korner, 1979; Schramm, 1982). The latter involves a graded somatotopic innervation of target cells, the establishment and maintenance of which has been extensively studied for the sympathetic outflow relayed by the superior cervical ganglion (see Purves and Lichtman, 1985 , for review). The graded innervation of the iterated set of thoracic paravertebral ganglia has received less attention, but is likely to involve similar developmental mechanisms. 

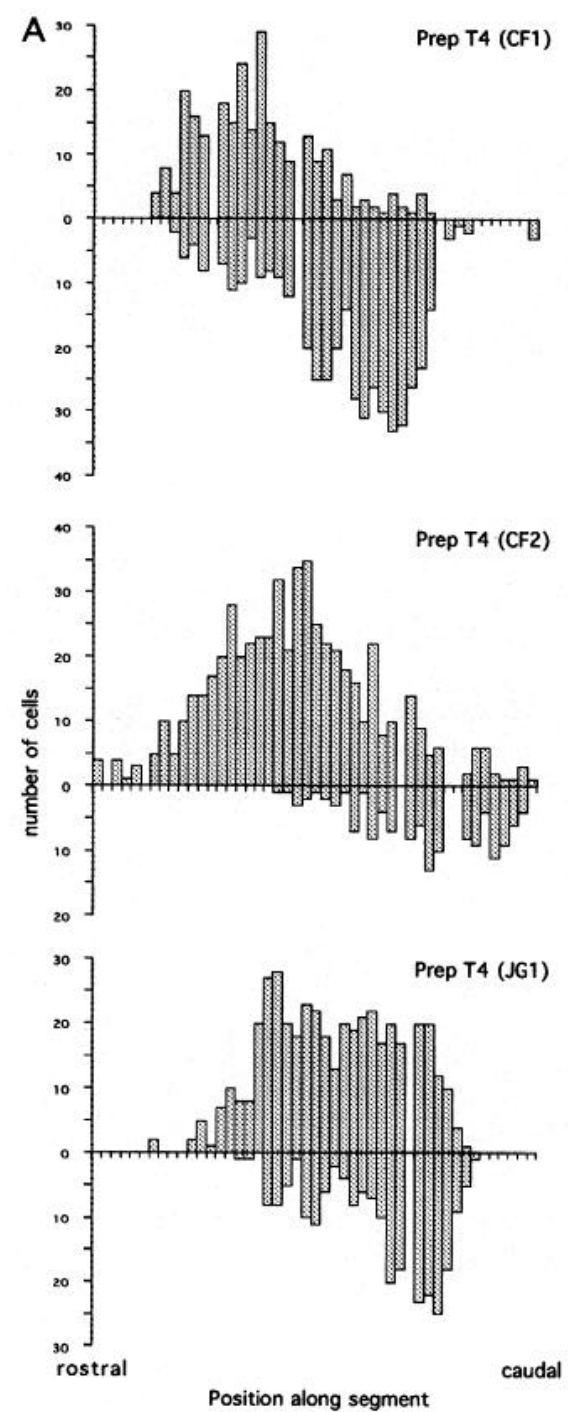

Position along segment
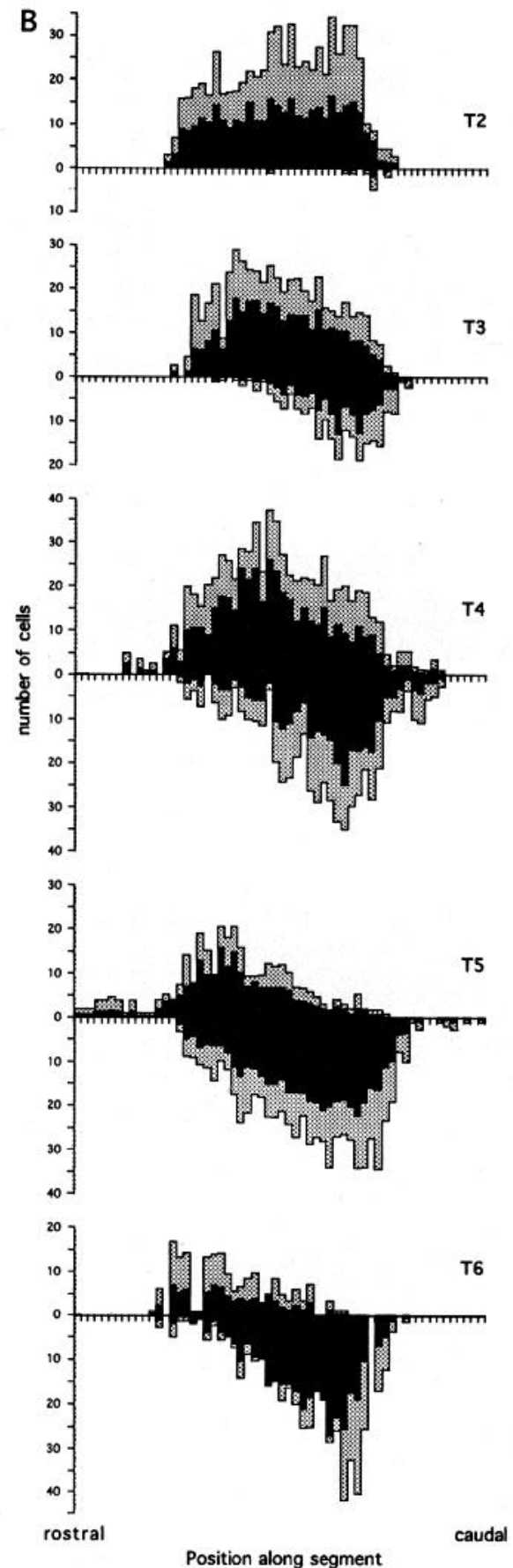

Figure 7. Relationship of preganglionic soma position to the direction of axon projection in segments T2-T6 of the E10 chicken. A, Total cell distributions for three individual preparations made at the T4 level. The number of cells projecting rostrally or caudally is plotted as a function of distance along the rostrocaudal axis of the segment. The bins represent data from consecutive $30 \mu \mathrm{m}$ sections through the spinal cord. Cells that project rostrally are shown above the zero axis while those that project caudally are shown below the zero axis. Missing bins represent lost or damaged sections from which cell counts were not possible. $B$, Similar histograms for segments T2-T6 are shown, with the mean indicated by the solid bars and the SDs represented by the shaded bars ( $n=3$ preparations at each level). The T4 histogram represents the three preparations shown in $A$.
Here, we have shown that individual sympathetic preganglionic neurons project only rostrally or caudally within the sympathetic chain, even though the spinal segment in which they reside provides innervation to both rostral and caudal ganglia. This is likely to be an important feature of sympathetic connectivity in the thoracic region. Bidirectional projections composed of separate rostrally and caudally projecting neuron populations allow for coordinate sympathetic activation in many contiguous segments without compromising the capability for selective activation in more restricted regions. That these separate populations are polarized within each segment may offer an advantageous developmental situation for the formation of specific connections from autonomic centers in the brainstem.

\section{How is the thoracic spinal cord organized?}

In both the rat and chicken, thoracic sympathetic preganglionic neurons are organized into discrete segmental units that exhibit an internal rostrocaudal polarity with respect to the direction of axon projection in the sympathetic chain. Moreover, the rostrocaudal bias of this internal polarity is graded from segment to segment along the longitudinal axis in the chicken embryo. The organization of the preganglionic projections thus includes (1) segmental periodicity, (2) intrasegmental gradients of neuronal identity, and (3) an axial gradient of segment identity. Since there is minimal overlap between thoracic segments, the transition from one segment to another entails a sharp change 


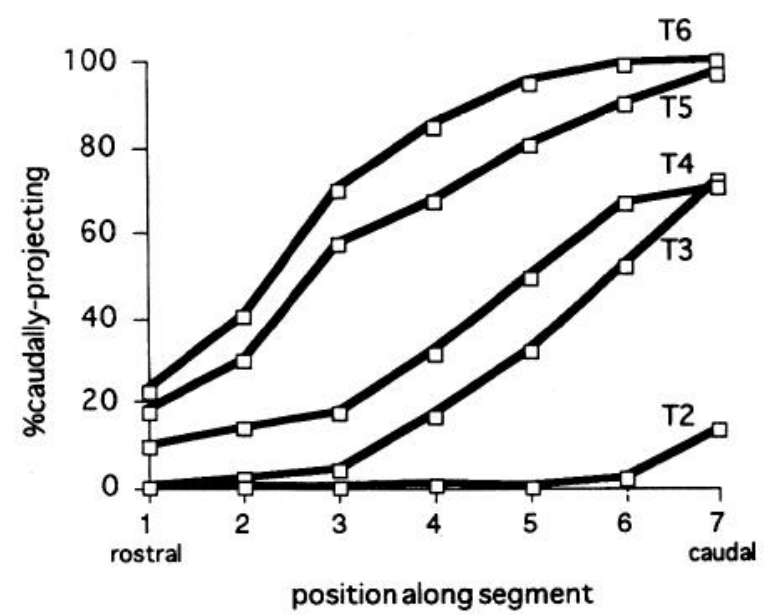

Figure 8 . The percentage of caudally projecting preganglionic neurons in segments T2-T6 as a function of distance along the rostrocaudal axis. In each segment, the percentage of caudally projecting neurons increases as a function of caudal position. The steepness of the function increases progressively between $\mathrm{T} 2$ and $\mathrm{T} 6$.

in the pathway choice of the preganglionic neurons. This segmentally iterated discontinuity in neuronal differentiation is the first demonstration of a segmental pattern of neuronal differentiation in the spinal cord of higher vertebrates.

In another study, Lim et al. (1991) used general and cellspecific markers to assay the spatial patterning of early neuronal differentiation in the chicken embryo. The lack of any obvious segmental patterns contrasts with the indisputable segmental patterns of neuronal differentiation in the spinal cord of lower vertebrates (Westerfield et al., 1986; Hanneman et al., 1988; Kuwada et al., 1990; see also references in Keynes and Lumsden, 1990), and in the chicken embryo's hindbrain (Glover, 1989; Lumsden and Keynes, 1989; Baker and Noden, 1990). Lim et al. (1991) suggest that the spinal cord of higher vertebrates may have lost an ancestral segmental organization in parallel with the evolution of limbs and the resultant need for intersegmental integration. Our findings suggest that the mechanisms underlying segmental patterns of differentiation are still present in the spinal cord of higher vertebrates. However, it may be that their overt expression is limited to the thoracic region, where an overriding intersegmental integration is expected to prevail the least.

Studies of the efferent connectivity of the spinal cord have revealed another feature of spinal cord organization, the presence of graded synaptic preferences. For example, when sympathetic preganglionic neurons are forced to compete for target cells of different segmental origins, they exhibit a preference for targets with coordinately ranked segmental origins (Mæhlen and Njå, 1981; Purves et al., 1981; Wigston and Sanes, 1982, 1985). Such findings have suggested the existence of gradients of positional information in the spinal cord and the periphery. Similar conclusions have been reached for somatic motoneurons that innervate large, multisegmental muscles. Here the spatial pattern of innervation is topographically represented within the motoneuron pool, suggesting a graded rostrocaudal matching of motoneuron and muscle fiber positions (Swett et al., 1970; Weeks and English, 1985; Laskowski and Sanes, 1987; Theriault and Diamond, 1988). Potential molecular correlates of the central and peripheral gradients suggested by patterns of target inner-
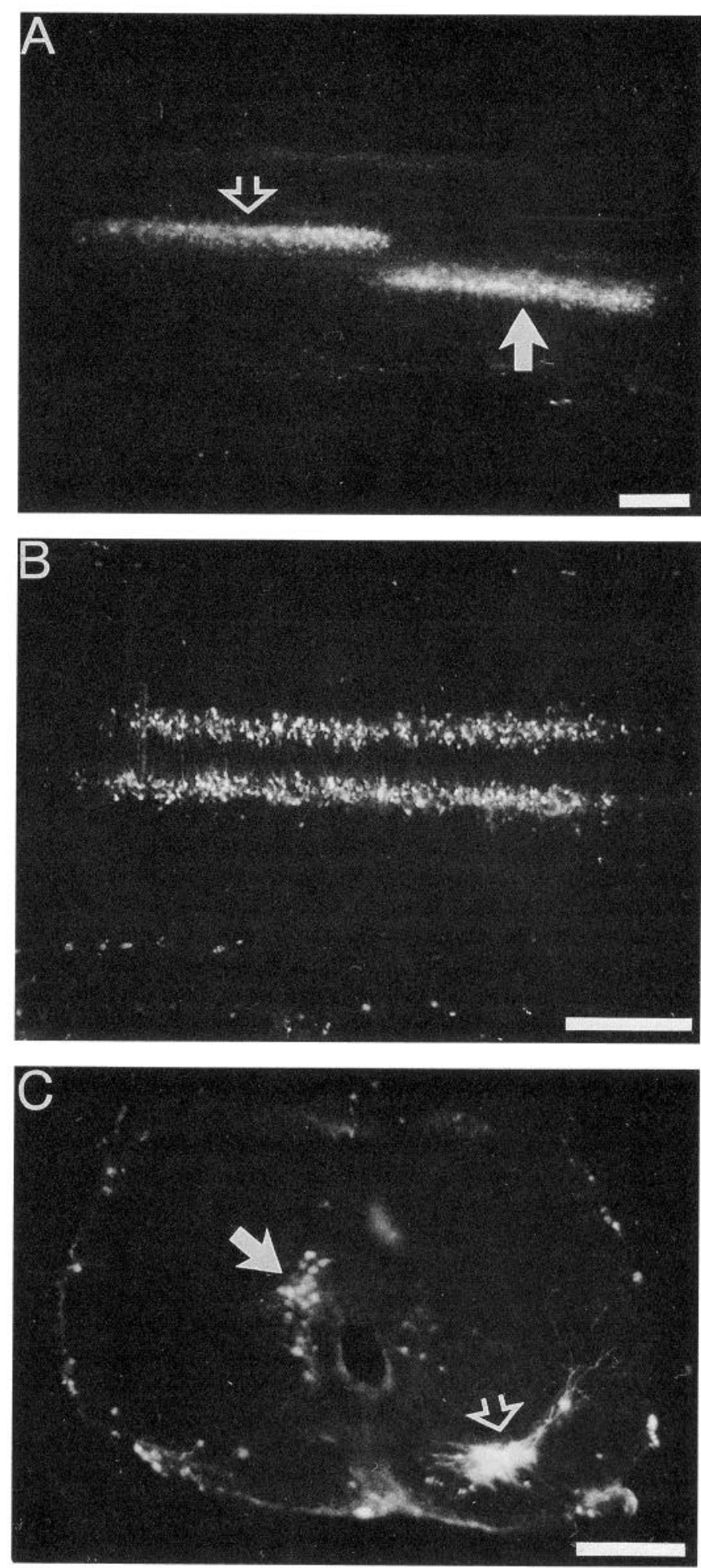

Figure 9. Segmental boundaries of preganglionic neurons in the E10 chicken. A, Segmental distribution of T3 (solid arrows) and T4 (open arrows) preganglionic neurons labeled with dextran amines from their respective chain ganglia (200 $\mu \mathrm{m}$ horizontal section). $B$, Symmetry of the distribution of left and right preganglionic neurons at T3 retrogradely labeled from the T3 sympathetic ganglion on each side $(200 \mu \mathrm{m}$ horizontal section). $C$, Transverse $30 \mu \mathrm{m}$ section showing T3 preganglionic neurons (solid arrow) and intercostal motoneurons (open arrow) retrogradely labeled from the T3 sympathetic ganglion or the T3 intercostal nerve, respectively. Scale bars, $200 \mu \mathrm{m}$. 


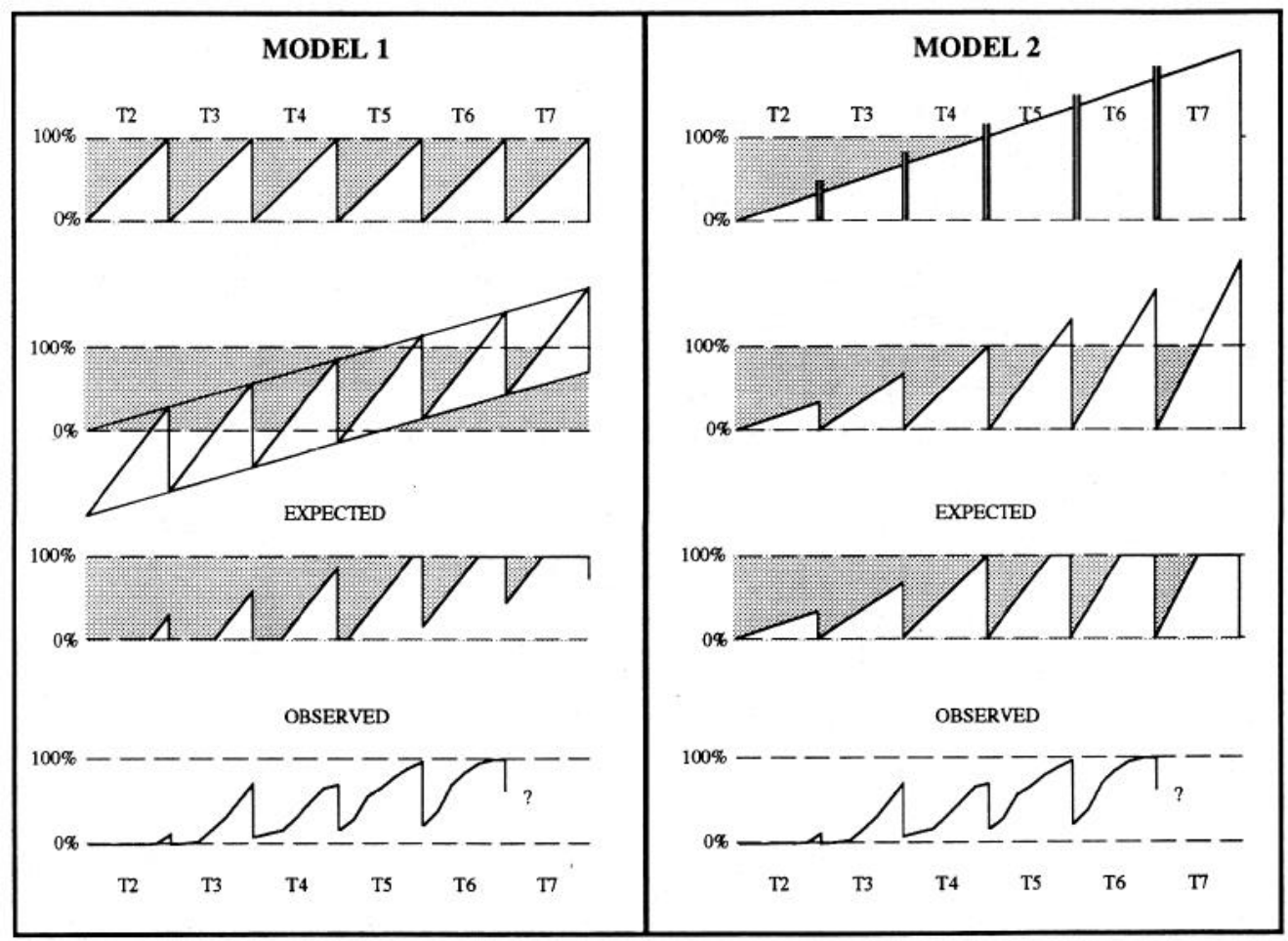

Figure 10. Two hypothetical models for generating a combined graded and segmented pattern of neuronal differentiation in the thoracic spinal cord. In model 1, the thoracic region is partitioned into equivalent segments, each with an intrinsically polarized longitudinal axis. The intrasegmental polarity is depicted as a linear gradient of a signal that specifies neurons to project caudally (producing from 0 to $100 \%$ caudally projecting neurons). Segmental iteration generates a sawtooth distribution of this signal (top panel). A multisegmental gradient is superimposed on this distribution and biases the initially equivalent intrasegmental gradients according to rostrocaudal position (second panel). By requiring individual neurons to exhibit a binary response to the signal according to appropriate threshold levels (indicated by 0 and $100 \%$ with intermediate values shaded in gray), the intrasegmental distributions of caudally projecting neurons acquire segment-specific profiles (third panel). The expected distribution is similar to the observed pattern (bottom panel). In model 2, the thoracic region contains a single multisegmental gradient of a signal that specifies neurons to project caudally (top panel). Axons exhibit an inherent tendency to repel each other, and the degree of repulsion is directly related to the difference in positional values the axons bear. The allocation of peripheral axons to segmental roots restricts the repulsive interactions to axons within a segmental set, such that axons with the minimum positional value within each segment are driven to project rostrally (second panel). Again, by imposing appropriate thresholds, the intrasegmental distributions of caudally projecting neurons acquire segment-specific profiles (third panel) that are similar to those observed (bottom panel).

vation include rostrocaudal gradients of Hox gene expression in the spinal neural tube (Gruss and Kessel, 1992), a Schwann cell antigen that is expressed in a rostrocaudal gradient in the sympathetic chain (Suzue et al., 1990), and a rostrocaudal gradient in the expression of muscle-specific transgenes (Donoghue et al., 1992; Grieshammer et al., 1992). Currently, there are no reports of a segmental modulation of these gradients.

\section{Potential pattern-generating mechanisms}

In attempting a mechanistic explanation for the observed pattern of sympathetic preganglionic outflow, it is crucial to determine whether the three pattern elements, namely, segmental periodicity, intrasegmental gradients of neuronal identity, and an axial gradient of segment identity, are established by the same or different mechanisms. Moreover, one must know the extent to which the underlying mechanisms have their sites of action centrally, within the spinal cord, or peripherally, in the tissues through which the preganglionic axons grow.

To illustrate the potential role of peripheral tissues in establishing the pattern, there is evidence that cues in the nascent sympathetic chain are involved in the directional choices of the preganglionic axons. Yip (1990) has transplanted thoracic spinal segments to ectopic levels in the chicken embryo and shown that preganglionic neurons project in the sympathetic chain in directions appropriate for their new rostrocaudal position. He suggests that the sympathetic chain bears different directional cues at different levels. Hence, preganglionic axons originating from the most rostral thoracic segments encounter only rostral cues and thus project only rostrally, those originating from the most caudal segments encounter only caudal cues and thus project only caudally, while those originating from intermediate thoracic segments encounter both rostral and caudal cues and thus project in both directions. Although this could easily generate the systematic shift from rostral to caudal predominance of projections originating from successively more caudal segments, it is unclear whether it could generate the observed internal polarity within each segment.

To illustrate the potential role of centrally operating factors in establishing the pattern, there is evidence that spinal cord neurons may be determined to recognize specific directional cues prior to axon outgrowth. The existence of positional signals that specify neuronal identity in the neural tube is well established (reviewed in Dodd, 1992; Wilson and Easter, 1992). In the zebrafish embryo, for example, the peripheral projection established by a primary motoneuron is determined, during a critical time period, by the position of the neuron along the rostrocaudal axis of the segment in which it resides (Eisen, 1991). A similar finding has recently been made in the chicken embryo (Matise 
and Lance-Joncs, 1992). Presumably, position-specific signals induce the expression of a specific set of receptors in the motoneuron's growth cone that then determine the subsequent choice of pathway and direction of growth. By the same token, preganglionic neurons could be induced as a function of position within their segment of origin to express differentially receptors for rostral and caudal cues in the sympathetic chain.

An attractive possibility is that all three attributes of the observed pattern of preganglionic axon outgrowth are specified by positional signals acting within the spinal cord itself. Figure 10 illustrates two models for how this could occur. The first involves a simple combination of two sets of positional signals, one specifying a global, multisegmental gradient, the other specifying a segmentally iterated "sawtooth" pattern (Fig. 10A). A multisegmental gradient could be provided by, for example, the graded Hox gene expression noted above, but what about the sawtooth pattern? The spinal neural tube bears segmentally iterated boundaries to cell migration (Stern et al., 1991) that generate lineage restrictions analogous to those represented by rhombomere boundaries in the hindbrain (Fraser et al., 1990). These partition the spinal cord into polyclonal segmental units that could become individually polarized along their rostrocaudal axes by cell-cell interactions, as occurs in the body segments of Drosophila (Peifer and Bejsovec, 1992). However, the segmentally iterated boundaries to cell migration are not in register with the segmental blocks of motoneurons, being out of phase by a quarter of the length of the block, at least at early embryonic stages (Stern et al., 1991). In this regard it is important to note that the intrasegmental patterns of differentiation in the thoracic sympathetic preganglionic column have been assayed at a much later stage than have the boundaries to cell migration. Ascertaining the spatial relationship between the two at cellular resolution will require their direct comparison in the same preparation, since there are no independent landmarks for segmental boundaries in the thoracic region of the cord that can be used at both time points.

The second model dispenses with the set of segmentally iterated positional signals, but requires that the effect of the global, multisegmental gradient is secondarily segmented by the formation of the ventral roots. Since the ventral roots force the preganglionic axons from different segments to enter the nascent sympathetic chain at different sites, each segmental contingent of preganglionic neurons may be operating effectively as an independent unit regarding the choice of rostral or caudal direction. Hence, positional differences among preganglionic neurons would be maximal for the extremes of each segment. This could drive neurons on opposite sides of a segment boundary to project in different directions despite experiencing nearly identical positional signals (Fig. 10B). In such a scheme, the transition from caudally projecting neurons to rostrally projecting neurons at segment boundaries would represent a discontinuity in axon interactions, rather than a discontinuity in positional information within the cord. Experiments to distinguish between the two models presented in Figure 10 are currently in progress.

To summarize, the results of this study show that individual chicken or rat sympathetic preganglionic neurons project only rostrally or caudally within the sympathetic chain, even though the spinal segment in which they reside provides innervation to both rostral and caudal ganglia. The preganglionic neurons are organized into discrete segmental units that exhibit an internal rostrocaudal polarity with respect to the direction of axon projection in the sympathetic chain. The rostrocaudal bias of this internal polarity is graded from segment to segment along the longitudinal axis. A major implication of these results is that the pattern of preganglionic projections may derive from an intrinsic segmentation of the spinal cord.

\section{References}

Baker R, Noden D (1990) Segmental organization of VIth nerve related motoneurons in the chick hindbrain. Soc Neurosci Abstr 16: 318

Cannon WB (1915) Bodily changes in pain, hunger, fear and rage. An account of recent researches into the function of emotional excitement. New York: Appleton.

Dodd J (1992) Mesodermal control of neural cell identity in vertebrates. Curr Opin Neurobiol 2:3-8.

Donoghue MJ, Morris-Valero R, Johnson YR, Merlie JP, Sanes JR (1992) Mammalian muscle cells bear a cell-autonomous, heritable memory of their rostrocaudal position. Cell 69:67-77.

Eisen J (1991) Determination of primary motoneuron identity in developing zebrafish embryos. Science 252:569-572.

Ezerman EB, Glover JC, Forehand CJ (1990) Segmental organization of thoracic preganglionic neurons in chick and rat embryos. Soc Neurosci Abstr 16:331.

Forehand CJ, Rubin E (1986) Specificity of sympathetic preganglionic projections: rat preganglionic neurons project to either rostral or caudal ganglia along the sympathetic chain. Soc Neurosci Abstr 12:1056.

Fraser S, Keynes R, Lumsden A (1990) Segmentation in the chick embryo hindbrain is defined by cell lineage restrictions. Nature 344 : $431-435$.

Gimlich RI, Braun J (1985) Improved fluorescent compounds for tracing cell lineage. Dev Biol 109:509-514.

Glover JC (1989) Rhombomeres and longitudinal boundaries in the chicken embryo brainstem subdivide vestibular neurons according to axonal pathway. Soc Neurosci Abstr 15:959.

Glover JC, Petursdottir G, Jansen JKS (1986) Fluorescent dextranamines used as axonal tracers in the nervous systern of the chicken embryo. J Neurosci Methods 18:243-254.

Grieshammer U, Sassoon D, Rosenthal N (1992) A transgene target for positional regulators marks early rostrocaudal specification of myogenic lineages. Cell 69:79-93.

Gruss P, Kessel M (1992) Axial specification in higher vertebrates. Curr Opin Genet Dev 1:204-210.

Guthrie S, Butcher M, Lumsden A (1991) Patterns of cell division and interkinetic nuclear migration in the chick embryo hindbrain. $J$ Neurobiol 22:742-754.

Hanneman E, Trevarrow B, Metcalfe WK, Kimmel CB, Westerfield M (1988) Segmental pattern of development of the hindbrain and spinal cord of the zebrafish embryo. Development 103:49-58.

Kessel M, Gruss P (1990) Murine developmental control genes. Science 249:374-379.

Keynes R, Lumsden A (1990) Segmentation and the origin of regional diversity in the vertebrate central nervous system. Neuron 2:1-9.

Keynes RJ, Stern CD (1988) Mechanisms of vertebrate segmentation. Development 103:413-429.

Korner PI (1979) Central nervous control of autonomic cardiovascular function. In: Handbook of physiology, Sec 2, Vol 1, The cardiovascular system (Berne RM, Sperelakis N, Geiger S, eds), pp 691-739. New York: American Physiological Society.

Kuwada JY, Bernhardt RR, Chitnis AB (1990) Pathfinding by identified growth cones in the spinal cord of zebrafish embryos. J Neurosci 10:1299-1308.

Lance-Jones C, Landmesser L (1981) Pathway selection by embryonic chick motoneurons in an experimentally altered environment. Proc R Soc Lond [Biol] 214:19-52.

Langley JN (1892) On the origin from the spinal cord of the cervical and upper thoracic sympathetic fibres, with some observations on white and grey rami communicantes. Philos Trans R Soc Lond [Biol] 183:85-124.

Langley JN (1894) The arrangement of the sympathetic nervous system, based chiefly on observations upon pilo-motor nerves. J Physiol (Lond) 15:176-244.

Laskowski MB, Sanes JR (1987) Topographic mapping of motor pools onto skeletal muscles. J Neurosci 7:252-260.

Lichtman JW, Purves D, Yip JW (1979) On the purpose of selective 
innervation of superior cervical ganglion cells. J Physiol (Lond) 292: 69-84.

Lichtman JW, Purves D, Yip JW (1980) Innervation of sympathetic neurones in the guinea-pig thoracic chain. J Physiol (Lond) 298:285299.

Lim TM, Jacques KF, Stern CD, Keynes RJ (1991) An evaluation of myelomeres and segmentation of the chick embryo spinal cord. Development 113:227-238.

I umsden A (1990) The cellular basis of segmentation in the developing hindbrain. Trends Neurosci 13:329-339.

Lumsden A, Keynes R (1989) Segmental patterns of neuronal development in the chick hindbrain. Nature 337:424-428.

Mæhlen J, Njå A (1981) Selective synapse formation during sprouting and after partial denervation of the guinea-pig superior cervical ganglion. J Physiol (Lond) 319:555-567.

Matise MP, Lance-Jones C (1992) The timing of motoneuron commitment in the developing chick spinal cord. Soc Neurosci Abstr 18: 1111 .

Narayanan CH, Hamburger V (1971) Motility in chick embryos with substitution of lumbosacral by brachial and brachial by lumbosacral spinal cord segments. J Exp Zool 178:415-432.

Njå A, Purves D (1977a) Specific innervation of guinea-pig superior cervical ganglion cells by preganglionic fibres arising from different levels of the spinal cord. J Physiol (Lond) 264:565-583.

$\mathrm{Njå} \mathrm{A,} \mathrm{Purves} \mathrm{D} \mathrm{(1977b)} \mathrm{Re-innervation} \mathrm{of} \mathrm{guinea-pig} \mathrm{superior} \mathrm{cer-}$ vical ganglion cells by preganglionic fibres arising from different levels of the spinal cord. J Physiol (Lond) 272:633-651.

Peifer M, Bejsovec A (1992) Knowing your neighbors: cell interactions determine intrasegmental patterning in Drosophila. Trends Genet $8: 243-249$.

Purves D, Lichtman JW (1985) Selective synaptic connections. In: Principles of neural development, pp 229-250. Sunderland, MA: Sinauer.

Purves D, Thompson W, Yip J (1981) Re-innervation of ganglia transplanted to the neck from different levels of the guinea-pig sympathetic chain. J Physiol (Lond) 313:49-63.

Rubin E (1985) Development of the rat superior cervical ganglion: ingrowth of preganglionic axons. J Neurosci 5:685-696.

Rubin E, Purves D (1980) Segmental organization of sympathetic preganglionic neurons in the mammalian spinal cord. J Comp Neurol 192:163-174.

Schramm LP (1982) Ganglionic, spinal and medullary substrates for functional specificity in circulatory regulation. In: Developments in neuroscience, Vol 15, Circulation, neurobiology, and behavior (Smith OA, Galosy RA, Weiss M, eds), pp 23-33. New York: Elsevier.

Stern CD, Jacques KF, Lim TM, Fraser SE, Keynes RJ (1991) Segmental lineage restrictions in the chick embryo spinal cord depend on the adjacent somites. Development 113:239-244.

Suzue T, Kaprelian Z, Patterson PH (1990) A monoclonal antibody that defines rostrocaudal gradients in the mammalian nervous system. Neuron 5:421-431.

Swett JE, Eldred E, Buchwald JS (1970) Somatotopic cord-to-muscle relations in efferent innervation of cat gastrocnemius. Am J Physiol 219:762-766.

Theriault E, Diamond J (1988) Intrinsic organization of the rat cutaneous trunci motor nucleus. J Neurophysiol 60:463-477.

Weeks OI, English AW (1985) Compartmentalization of the lateral gastrocnemius motor nucleus. J Comp Neurol 235:255-267.

Westerfield M, McMurray J, Eisen JS (1986) Identified motoneurons and their innervation of axial muscles in the zebrafish. J Neurosci 6:2267-2277.

Wigston DI, Sanes JR (1982) Selective reinnervation of adult mammalian muscle by axons from different segmental levels. Nature 299: 464-467.

Wigston DJ, Sanes JR (1985) Selective reinnervation of intercostal muscles transplanted from different segmental levels to a common site. J Neurosci 5:1208-1221.

Wilkinson DG, Krumlauf R (1990) Molecular approaches to the segmentation of the hindbrain. Trends Neurosci 13:335-339.

Wilson SW, Easter SS (1992) Acquisition of regional and cellular identities in the developing zebrafish nervous system. Curr Opin Neurobiol 2:9-15.

Yip JW (1986) Specific innervation of neurons in the paravertebral sympathetic ganglia of the chick. J Neurosci 6:3459-3464.

Yip JW (1990) Identification of location and timing of guidance cues in sympathetic preganglionic axons of the chick. J Neurosci 10:24762484 . 